\title{
Expression of the Toll-like receptors 2 gene as a genetic determinant of the formation of newborns multiple organ failure syndrome
}

\author{
Dmytro A. Shkurupii \\ Department of Anesthesiology and Intensive Care of Ukrainian Medical Stomatological Academy, Poltava, Ukraine. \\ E-mail:d.a.shkurupiy@gmail.com
}

Received: 20th November 2017, Revised: 24th December 2018, Accepted: 4th January 2019

SUMMARY: Shkurupii DA. Expression of the Toll-like receptors 2 gene as a genetic determinant of the formation of newborns multiple organ failure syndrome. Turk J Pediatr 2019; 61: 500-504.

Activation of the humoral immune response in newborns' multiple organ failure syndrome is associated with the production of interleukin-1 $\beta$, which is recognized via Toll-like receptors 2 (TLR2). The aim of this study was to determine the role of expression of the Toll-like receptors 2 gene in the pathogenesis of newborns with multiple organ failure syndrome.

A prospective observational cohort study of 149 newborns was conducted. The main group $(n=113)$ were newborns with multiple organ failure syndrome, whereas the comparison group $(n=36)$ was newborns without this syndrome. The study included analysis of the expression of the TLR2 gene and its comparison with the concentration of interleukin-1 $\beta$, peripheral blood lymphocyte count and clinical signs of the course of the disease.

It was found that the population risk of reducing TLR2 expression was $79.33 \%$ in the main group, and $27.62 \%$ in the comparison group. Increasing the expression of the gene TLR2 in newborns leads to an increase in the concentration of interleukin-1 $\beta$, an increase in the level of peripheral blood lymphocytes and is associated with the formation of microbial loci. Expression reduction of the TLR2 gene may lead to the development of multiple organ failure syndrome, systemic inflammatory response syndrome, increase in the number of affected organs, increased frequency of involvement in the immune system, and the need for aggressive respiratory therapy.

Key words: multiple organ failure, toll-like receptor 2, newborn.

According to the World Health Organization, about $46 \%$ of all deaths of children under the age of five are newborns. Of these, $80 \%$ are newborns with low body weight and gestational age, perinatal infections, birth asphyxia, and birth trauma. ${ }^{1}$ Improving the quality of prosthetic repair of vital functions in critical newborn infants has helped to stretch the classical phases of tanatogenesis in time, leading to the formation of a specific condition known as "multiple organ failure syndrome" (MOFS). ${ }^{2}$

Analyzed mainly on adult patients, MOFS is considered a universal lesion of several organs and systems, mainly due to bacterial translocation and uncontrolled cytokine, peroxide, endothelial reactions. ${ }^{3}$ In newborns, the development of this syndrome has its own peculiarities associated with the formation of intrauterine fetal inflammatory response, microbiota of the organism, and adaptation to extrauterine existence. ${ }^{4}$

Activation of the humoral inflammatory response is associated with the production of cytokines, in particular interleukin $1 \beta$ (IL$1 \beta$ ), which is the trigger of proinflammatory cytokine reactions. ${ }^{5}$ Its production is realized by activation of Toll-like receptors 2 (TLR2) and is regulated by the corresponding gene. 
There is evidence of the predetermining role of these gene mechanisms in the features of the clinical course of critical conditions in newborns. ${ }^{6}$ In this regard, it can be assumed that there is a genetic determination of the development of MOFS in neonatology.

Aim of this work: To determine the role of expression of the TLR2 gene in the pathogenesis of MOFS.

\section{Material and Methods}

\section{Study population}

A prospective observational cohort study of 149 newborns aged 0 to 27 days, who were on treatment for the effects of perinatal asphyxiation and perinatal infection, was conducted. The main group $(n=113)$ consisted of newborns with signs of MOFS, who underwent intensive therapy. The comparison group $(\mathrm{n}=36)$ consisted of newborns who had no signs of MOFS, did not need intensive care and were on treatment in the departments of pathology and nursing of newborns.

Prior to the start of the study, informed consent was obtained from the legal representatives of the newborns and the permission from the commission on ethical issues and bioethics above that institution (study was approved 19 Feb 2008, the report number 57). During the study, the patients' rights were respected in accordance with the requirements of the 1975 Helsinki Declaration, as amended in 2005.

\section{Clinic Methods}

MOFS was recorded with simultaneous presence of disorders of two or more organs or systems according to the author's scale. ${ }^{7}$

To determine the risk of bacterial aggression in hospitals, the Device Utilization Ratio (DURindex) was calculated, which is the ratio of the sum of days of use of the endotracheal tube, central venous access and urinary catheter to the number of days spent in the treatment facility, the ward. ${ }^{8}$

Signs of the systemic inflammatory response syndrome (SIRS) were also registered..$^{9}$

\section{Laboratory Methods}

Laboratory studies were conducted on the basis of the Research Institute of Genetic and Immunological Foundations of Development of Pathology and Pharmacogenetics of the Higher State Educational Institution of Ukraine "Ukrainian Medical Dental Academy". The study included analysis of TLR2 gene expression and its comparison with IL-1 $\beta$ concentration, peripheral blood lymphocyte count and clinical signs of MOFS flow.

The expression level of the TLR2 gene was determined by polymerase chain reaction, reverse transcription using the RIBO-sol-B reagent kit ("AmpliSens", Russia) in the presence of the SYBR Green I dye. The $\beta$-actin gene was used as the reference gene.

The concentration of IL- $1 \beta$ in the neonatal plasma was determined using a test system for enzyme-linked immunosorbent assays in accordance with the manufacturer's protocols ("Cytokine", Russia).

The level of peripheral blood lymphocytes was determined by a unified method of counting in automatic hematology analysers.

In addition, in newborns with MOFS, after 5 days, the dynamic orientation of changes in peripheral blood lymphocyte levels, the concentration of IL-1 $\beta$ in blood plasma, and the microbial flora of microbial loci were recorded (oropharynx, bronchial mucus, ocular gaps, auricles, umbilical remainder, rectum), and a comparison was made between these and the direction of expression of the TLR2 gene by correlation analysis.

\section{Statistical Analysis}

Statistical analysis was carried out using Microsoft Office Excel 2003 software, using methods of descriptive statistics and nonparametric criteria. The absolute values of the indicators, the number of studies (n), the median $(\mathrm{Me})$, the quarter-scale (50L 50U), the Wilcoxon-Mann-Whitney criterion (U), Spearman's correlation coefficient (R), Pearson's $\chi^{2}$ criterion, the critical significance level of the error probability of the first kind (p) were used. For the population extrapolation of the risk of detecting the genetic determination of MOFS, the index of normalization of intensive indicators was applied. 


\section{Results}

The results of genetic and immunological tests in the study groups are presented in the Table I.

Analysis of expression of the TLR2 gene with respect to the expression of the efferent $\beta$-actin gene showed that in the main group it was less; in the comparison group, it was more. The incidence of inhibition of TLR2 gene expression was different in the study groups and comprised 17 cases $(62.96 \%)$ and 8 cases $(33.33 \%)$ for the comparison group $(\chi 2=4.46, \mathrm{p}=0.03)$. Since the decrease in TLR2 expression was predominant in the neonatal group with MOFS, the population risk of its implementation was calculated for this factor. After the determination of the index of intensity indexation, which was 0.74 for the primary and 0.71 for the control group, the risk of revealing cases of inhibition of the expression of the TLR2 gene in the population among the newborns with MOFS was $79.33 \%$, and in newborns without MOFS it was 27, $62 \%(\chi 2=33.28, \mathrm{p}=0.001)$.

The concentration of IL-1 $\beta$ in plasma of newborns born with MOFS was somewhat lower in newborns without MOFS. However, there was no reliable statistical difference between these indices. The correlation analysis, on the other hand, revealed an inverse relationship between the expression of the TLR 2 gene and the content of IL-1 $\beta$ in the plasma of newborns with MOFS at the beginning of the study $(\mathrm{R}=-0.50 ; \mathrm{p}=$ $0.007 ; \mathrm{n}=27$ ). A direct correlation was also established between the expression of the TLR2 gene and the positive dynamics of IL-1 $\beta$ in the plasma of newborns with MOFS at the end of the study $(\mathrm{R}=0.40 ; \mathrm{p}=0.04 ; \mathrm{n}=27)$.
The level of lymphocytes in neonates with MOFS was lower than in newborns without this syndrome. Correlation analysis in neonates with MOFS showed a direct relationship between the expression of the TLR2 gene and the positive dynamics of peripheral blood lymphocytes $(\mathrm{R}=0.48, \mathrm{p}=0.02, \mathrm{n}=23)$.

Studies of the microbial colonization of newborns revealed bacterial contamination in 82 cases $(72.57 \%)$ in the baseline and in 23 cases $(63.89 \%)$ in the comparative groups $\left(\chi^{2}=0.99, \mathrm{p}=0.32\right)$. There was a direct correlation between the expression of the TLR2 gene and positive microbiological studies at the end of the study $(\mathrm{R}=0.52 ; \mathrm{p}$ $=0.07 ; \mathrm{n}=25)$. In neonates without MOFS, central venous access, intubation of the trachea and catheterization of the bladder were never used, so they had a DUR index of zero. In newborns with MOFS, the DUR-index was $1.06 \pm 0.08$ conventional units. Correlation analysis showed an inverse correlation of the DUR index with the expression of the TLR2 gene $(R=-0.33, p=0.02, n=51)$.

An inverse statistical relationship was found between some clinical manifestations of MOFS with the expression of the TLR2 gene, in particular:

- with the presence of MOFS $(\mathrm{R}=-0.30, \mathrm{p}=0.04, \mathrm{n}=51)$;

- with the implementation of the SIRS $(\mathrm{R}=-0.30, \mathrm{p}=0.04, \mathrm{n}=51)$;

- with number of affected organs $(\mathrm{R}=-0.33, \mathrm{p}=0.02, \mathrm{n}=51)$;

- with the frequency of involvement in the MOFS immune system $(\mathrm{R}=-0.34, \mathrm{p}=0.01, \mathrm{n}=51)$;

Table I. The Results of Genetic and Immunological Tests in Study Groups.

\begin{tabular}{llccccc}
\hline Indicator & Groups & $\mathrm{n}$ & $\mathrm{Me}$ & $50 \mathrm{~L}-50 \mathrm{U}$ & $\mathrm{U}$ & $\mathrm{p}$ \\
\hline $\begin{array}{l}\text { Expression of the TLR2 with } \\
\text { respect to the expression of the }\end{array}$ & Main & 27 & -1.25 & $-1.7-1.4$ & \multirow{2}{*}{255} & 0.2 \\
efferent $\beta$-actin gene (times) & Comparative & 24 & 1.1 & $-1.4-1.7$ & & \\
Concentration of IL-1 $\beta(\mathrm{pg} / \mathrm{ml})$ & Main & 80 & 221.8 & $60-375.6$ & \multirow{2}{*}{481} & 0.67 \\
& Comparative & 13 & 181.8 & $33.3-593.5$ & & \\
Level of lymphocytes (\%) & Main & 103 & 344 & $21.1-43$ & \multirow{2}{*}{608} & $<0.001$ \\
& Comparative & 110 & 52 & $44-60$ & & \\
\hline
\end{tabular}


- using non-invasive methods of respiratory support $(\mathrm{R}=-0.30, \mathrm{p}=0.04, \mathrm{n}=51)$.

\section{Discussion}

The results of the study indicate a significant influence of the orientation of TLR2 gene expression on the formation and clinical course of MOFS in newborns. A high percentage of the population's risk of reducing the expression of this gene makes it a marker for the formation of MOFS in this patient population.

As a result of the expression of the TLR2 gene, the synthesis of IL-1 $\beta$, which is the inducer of the proinflammatory cytokine cascade, is activated. In neonates with MOFS, a decrease in this interleukin is directly associated with a low expression of the TLR2 gene. Positive dynamics in the expression of this gene and, accordingly, IL-1 $\beta$, is observed only with time, which can be related both to the ability of the interleukin itself to support the expression of the TLR2 gene ${ }^{10}$ and the colonization of previously sterile microbial locus in the newborn's body, including - with the participation of aggressive flora of intensive care units. This is indirectly evidenced by an inverse correlation at the end of the study between the expression of the TLR2 gene and the DUR index: Increasing the expression of this gene led to an increase in inflammatory responses, which apparently made it necessary to reduce the use of invasive procedures to reduce microbial contamination.

An inadequate humoral response of newborns with MOFS is also indicated by a low level of lymphocytes, which is increasing due to an increase in the expression of the TLR2 gene. In the early neonatal period, physiological neutrophilia prevails, which creates relative lymphopenia. ${ }^{11}$ However, given the same age of the newborns under study, it can be stated that they develop lymphopenia associated with MOFS. It is known that IL- $1 \beta$ stimulates the proliferation of lymphocytes in the thymus and spleen and increases their functional activity of lymphocytes ${ }^{12}$, which explains the relationship of their level to the expression of the TLR2 gene.
A feature of newborns, probably again associated with the initial low expression of the gene TLR2, is an increase in this background of the number of affected organs and systems, signs of involvement in the process of the immune system, the development of the MOFS and SIRS. One of the signs of the latter is the neutrophil shift to the left. Obviously, in conditions of lymphocytopenia and depression of the humoral immune link, cellular immunity associated with the SIRS clinic and determining the severity of the patient's condition is activated, as indicated by the frequency of the use of aggressive techniques of respiratory therapy in conditions of reduced expression of the TLR2 gene.

Thus, in neonates under conditions of initially reduced expression of the TLR2 gene, its increase with further activation of IL-1 $\beta$ production can be regarded as a compensatory reaction. In this case, with the expressed expression of this gene, the hyperactivity of pro-inflammatory cytokines with the formation of the flow of SIRS and MOFS by the classical type is not excluded.

So, in newborns with MOFS, there is a tendency to decrease the expression of the TLR2 gene with respect to the expression of the efferent $\beta$-actin gene. The risk of detection of cases of oppression of this gene among newborns with MOFS was $79.33 \%$, in newborns without MOFS was $27.62 \%$. Amplifying the expression of the gene TLR2 in newborns leads to an increase in the concentration of IL- $1 \beta$ and associated with the formation of microbial locuses. Newborns with MOFS, in comparison with newborns without such a syndrome, have a low content of peripheral blood lymphocytes. Its increase is directly dependent on the expression of the TLR2 gene. In such newborns, the increase in the number of affected organs and systems, a frequency of signs of involvement in the process of the immune system, the development of MOFS itself, SIRS, and the use of aggressive methods of respiratory therapy are associated. In newborns with MOFS under conditions of initially reduced expression of the TLR2 gene, its further increase with the activation of IL-1 $\beta$ production can be regarded as a compensatory reaction. 


\section{REFERENCES}

1. WHO. Newborns: reducing mortality: Updated. 2017 [Electronic resourse].- Access mode: http://www. who.int/mediacentre/factsheets/fs333/en/.

2. Aufieri R, Picone S, Paolillo P. Multiple organ failure in the newborn. JPNIM 2014; 3(2) [Electronic resourse].- Access mode: http://www.jpnim.com/ index.php/jpnim/article/view/030254/217/.

3. Deitch EA. Gut-Origin sepsis: evolution of a concept. Surgeon 2012; 10: 350-356.

4. Hofer N, Kothari R, Morris N, Müller W, Resch B. The fetal inflammatory response syndrome is a risk factor for morbidity in preterm neonates. Am J Obstet Gynecol 2013; 209: 542.e1-542.e11.

5. Lukens JR, Gross JM, Kanneganti T-D. IL-1 family cytokines trigger sterile inflammatory disease. Front Immunol 2012; 3: 315.

6. Kholod D, Shkurupii D, Sonnik E. Immune changes in newborn infants with gastrointestinal failure requiring intensive care. Georgian Med News 2016; 7-8: 62-66.
7. Shkurupiy D. Clinical-morphological comparisons possible diagnostic method of multiple-organ-failure syndrome in newborns. Adv Sci J 2011; 2: 133135.

8. Fakih MG, Gould CV, Trautner BW, et al. Beyond infection: Device utilization ratio as a performance measure for urinary catheter harm. Infect Control Hosp Epidemiol 2016; 37: 327-333.

9. Hofer N, Müller W, Resch B. Systemic inflammatory response syndrome (SIRS) definition and correlation with early-onset bacterial infection of the newborn. Arch Dis Child Fetal Neonatal Ed 2010; 95: F151.

10. Matsumura $\mathrm{T}$, Ito $\mathrm{A}$, Takii $\mathrm{T}$, Hayashi $\mathrm{H}$, Onozaki $\mathrm{K}$. Endotoxin and cytokine regulation of toll-like receptor (TLR) 2 and TLR4 gene expression in murine liver and hepatocytes. J Interferon Cytokine Res 2004; 20: 915-921.

11. Parvez Y, Mathew AG. Hyperleukocytosis in newborn: A diagnosis of concern. Indian J Hematol Blood Transfus 2014; 30(Suppl 1): 131-132.

12. Sims JE, Smith DE. The IL-1 family: regulators of immunity. Nat Rev Immunol 2010; 10: 89-102. 